\title{
Bioinformatic approaches towards identification of potential repurposable drugs for COVID-19
}

\section{Manisha Mandal}

MGM Medical College, Kishanganj-855107, India https://orcid.org/0000-0002-9562-5534

Shyamapada Mandal ( $\nabla$ samtropmed@gmail.com )

University of Gour Banga, Malda-732103, India https://orcid.org/0000-0002-9488-3523

\section{Research Article}

Keywords: COVID-19, SARS-CoV-2, GSE 147507 dataset, protein-protein interaction, gene-drug interaction, repurposable drugs

Posted Date: July 10th, 2020

DOl: https://doi.org/10.21203/rs.3.rs-38164/v2

License: (1) This work is licensed under a Creative Commons Attribution 4.0 International License. Read Full License 


\section{Abstract}

Repurposing existing drugs approved for other conditions is crucial to identifying specific therapeutics against SARS-CoV-2 causing COVID-19 (coronavirus disease-2019) pandemic. Towards this attempt, it is important to understand how this virus hijacks the host system during the course of infection and determine potential virus- and host-targeted inhibitors. This study elucidates the underlying virus-host interaction based on differentially expressed gene profiling, functional enrichment and pathway analysis, protein-protein and protein-drug interactions utilizing the information on transcriptional response to SARS-CoV-2 infection from GSE 147507 dataset containing COVID-19 case relative to healthy control and infected cell culture compared to uninfected one. Low IFN signaling, chemokines level elevation, and proinflammatory cytokines release were observed markedly. We identified MYC-rapamycin and ABCG2rapamycin interactions, and unique gene signatures in case (regulation of protein modification and MAPK signaling) as well as in cell (metabolic dysregulation and interferon signaling) different from known COVID-19 genes.

\section{Highlights}

- Low IFN signaling, chemokines level elevation, and proinflammatory cytokines release were observed markedly

- Cyclosporine, doxycycline, chloroquine, rapamycin interacted with MYC, PPP3R1, FOS, ABCG2, TNF in case and culture

- Cyclosporine, azithromycin, rapamycin, oseltamivir interacted with IL6, CD8A, CD40LG, IL10, CSF3 known COVID-19 genes

- Chloroquine, lopinavir, ritonavir, interferon beta-1a antagonist are included in WHO solidarity trial for COVID-19

- Among a plethora of repurposable drugs those appearing here with unique gene signatures might be helpful in COVID-19

\section{Abbreviations}

ABCG2: ATP binding cassette subfamily G member 2,ACE2: angiotensin-converting enzyme 2, AHR: aryl hydrocarbon receptor, ALB: albumin, ALDH3A1: aldehyde dehydrogenase 3 family member A1, ACDH3A2: aldehyde dehydrogenase 3 family member A1, APOH: apolipoprotein $\mathrm{H}, \mathrm{ARG}$ : arginase 1, ATG: autophagy related genes, BATF2: basic leucine zipper ATF-like transcription factor 2, BPI: bactericidal permeability increasing protein, CASP5: apoptosis-related cysteine peptidase, CAT: catalase, CCL: chemokine ligand, CCR: chemokine receptor, CD: cluster of differentiation, CD8A: T-cell surface glycoprotein CD8 alpha chain,CD40LG: CD40 Ligand, CDK: cyclin dependent kinase, CES1:

carboxylesterase 1, CSF3: colony stimulating factor 3, CTSD: cathepsin D, CXCL: C-X-C motif chemokine ligand, CXCR: C-X-C motif chemokine receptor, CX3CL: C-X3-C motif chemokine ligand, CX3CR: C-X3-C motif chemokine receptor, CYP1A1: cytochrome P450 family 1 subfamily A member 1, DDX1: DEAD-box 
helicase 1, DEFA: defensin alpha, DUB: deubiquitination, DUSP1: dual-specificity phosphatase 1, E: envelope protein, EGR1: early growth response 1, ENPEP: glutamyl aminopeptidase, EPHX1: epoxide hydrolase 1,FCGR3A: Fc fragment of IgG receptor Illa, FOS: Fos Proto-Oncogene, FURIN: paired basic amino acid cleaving enzyme, GSTA1: glutathione S-transferase alpha 1, HLA-DRA: HLA class II histocompatibility antigen DR alpha chain, HLA-DBR: HLA class II histocompatibility antigen DR beta chain, HSPA8: heat shock protein family A (Hsp70) member 8, IFI: interferon alpha inducible protein, IFI44L: interferon induced protein 44 like, IFIT: interferon induced protein with tetratricopeptide repeats, IFITM: interferon induced transmembrane protein, IFN: interferon, IFNA: interferon alpha, IFNB: interferon beta, IFNB1: interferon beta 1, IFNG: interferon gamma, IL: interleukin, IL1B: interleukin 1 beta, IL1R2: interleukin 1 receptor type 2, IL18RAP: interleukin 18 receptor accessory protein, IL2RA: interleukin 2 receptor alpha, IRF: Interferon Regulator Factor, ISG: Interferon Stimulated Gene, ISRE: IFN-stimulated response element, JAK1: janus kinase 1, JUNB: JunB proto-oncogene AP-1 transcription factor subunit, LC3: microtubule-associated protein 1A/1B-light chain 3, M: membrane protein, MAPK: mitogen-activated protein kinases, MFN2: mitofusin 2, MKI67: marker of proliferation Ki-67, MMP8: matrix

metallopeptidase 8, MPO: myeloperoxidase, MX: MX dynamin like GTPase 1, MYC: MYC proto-oncogene, $\mathrm{N}$ : nucleocapsid protein, NendoU: uridylate-specific endoribonuclease, NFATC3: nuclear factor of activated T cells 3, NFKB: Nuclear Factor Kappa B, NKRF: NFKB Repressing Factor, NLRP3: NLR family pyrin domain containing 3, nsp3/PL2-PRO: papain like protease, nsp5/MPRO: main protease, nsp12/RdRp: RNA dependent RNA polymerase, nsp7-nsp8: primase complex, nsp13: helicase, nsp14: exoribonuclease, nsp15: endonuclease, OAS1: 2'-5'-oligoadenylate synthetase 1, OASL: 2'-5'oligoadenylate synthetase like, ORF: open reading frame, PDGFRB: platelet derived growth factor receptor beta, PE: phosphatidyl ethanolamine, PIAS1: protein inhibitor of activated STAT1, PKR: protein kinase R, PLEKHA4: pleckstrin homology domain containing A4, PPP3R1: protein phosphatase 3 regulatory subunit B alpha, PRR: Pattern Recognition Receptor, p-STAT1: phosphorylated signal transducers and activators of transcription, RBD: receptor-binding domain, RPS6: ribosomal protein S6, RSAD2: Radical s-adenosyl methionine domain containing 2, RTC: replicase-transcriptase complex, S: surface spike protein, SFN: epithelial cell marker protein 1, S100A9: S100 calcium binding protein A9, TF: transferring, TMPRSS2: transmembrane serine protease 2, TNF: tumor necrosis factor, TNFSF10: TNF superfamily member 10, TNNI3: troponin 13, TSC1: tuberous sclerosis 1, ULK: Unc-51 like autophagy activating kinase, XAF1: Xlinked inhibitor of apoptosis protein associated factor 1.

\section{Introduction}

As of June 25, 2020, the ongoing COVID-19 (coronavirus disease-2019) pandemic caused 9,296,202 people infections globally including 479,133 deaths (WHO, 2020), because of the lack of specific treatments or vaccines. Repurposing drugs may possibly curtail the time and costs compared to de novo drug discovery, in addition to providing principal information on pharmacology and toxicology, as well as ascertaining novel indications for prompt clinical trials and regulatory assessment (Shaha et al., 2020; Gordon et al., 2020; Guy et al., 2020). The present study address this by mapping the interaction between viral proteins and human proteins in SARS-CoV-2 infected case compared to healthy control, in SARS- 
CoV-2 infected- compared to uninfected cell culture. This was achieved by downloading the GSE 147507 dataset from the Gene Expression Omnibus (GEO) database (www.ncbi.nlm.nih.gov/geo/) related to transcriptional response to SARS-CoV-2 infection (Blanco-Melo et al., 2020). The underlying virus-host interactions was elucidated based on differentially expressed genes (DEGs) profiling, functional enrichment and pathway analysis, protein-protein interaction (PPI) network, and protein-drug interaction (PDI) in context to COVID-19. The current study explore the major regulatory genes as potential biomarkers of COVID-19, transcriptional response to SARS-CoV-2 infection in cell culture as well as in case, comparison of the known gene signatures in COVID-19 with that in case and culture, and identification of potential repurposable drugs for COVID-19 treatment.

\section{Methods}

\subsection{COVID-19 disease associated genes and network}

The top 100 COVID-19 associated human genes were retrieved from disease query of the STRING (Search Tool for the Retrieval of Interacting Genes) (http://string-db.org/cgi/input.pl; version 1.5.0) App (Doncheva et al., 2019) of the Cytoscape software, version 3.7.2 (https://cytoscape.org/), according to Shannon et al. (2003). A STRING network was created with top 44 COVID-19 related genes ranked in order of disease scores and degree connectivity; the set of top 44 genes were named as group $A$ with their disease scores and degree connectivity ranging from 1.043351 to 3.922025 and from 1 to 29 , respectively.

\subsection{SARS-CoV-2 gene expression data acquisition and protein- protein interaction network analysis}

The gene expression dataset GSE5147507 was obtained from the gene expression omnibus database (www.ncbi.nlm.nih.gov/geo/) related to transcriptional response to SARS-CoV-2 infection (Blanco-Melo et al., 2020), from where 10 samples were selected and divided into group B and group C. Group B contained technical replicates of human lung biopsy samples from a single COVID-19 deceased male patient of $>60$ years with no treatment (GSM4462415: Series15_COVID19Lung_1 and GSM4462416: Series15_COVID19Lung_2) and biological replicates of human lung biopsy samples derived from two uninfected male patients of $>60$ years with no treatment (GSM4462413: Series15_HealthyLungBiopsy_1 and GSM4462414: Series15_HealthyLungBiopsy_2). Group C contained independent biological triplicates

of transformed lung alveolar cells (A549) transduced with a vector expressing human ACE2 mock treated (GSM4486157: Series16_A549-ACE2_Mock_1, GSM4486158: Series16_A549-ACE2_Mock_2, and GSM4486159: Series16_A549-ACE2_Mock_3) or infected with USA-WA1/2020 SARS-CoV-2 strain (GSM4486160: Series16_A549-ACE2_SARS-CoV-2_1, GSM4486161: Series16_A549-ACE2_SARS-CoV2_2, and GSM4486162: Series16_A549-ACE2_SARS-CoV-2_3). 
The DEGswere identified in COVID-19 case relative to the uninfected control (group B), and in ACE2induced A549 cell line with SARS-CoV-2 infection compared to the uninfected cell line (group C); the threshold for DEGs were set as $|\log 2 F o l d C h a n g e|\left(\left|\log _{2} F C\right|\right)>10$, and $\left|\log _{2} F C\right|>3$ respectively, for the two groups. A PPI network was constructed with the DEGs in group B and group C using STRING App of the Cytoscape software, version 3.7.2 (https://cytoscape.org/), as per Shannon et al. (2003). The significant DEGs were expressed as heatmaps using R programming (https://www.r-project.org/; version 3.6.1 [2019-07-05]) gplots tools from the Biobase packages (https://CRAN.R-project.org/package = gplots) of the Bioconductor project (Huber et al., 2015).

\subsection{GO and KEGG pathway enrichment analyses}

The DEGs $\| \log _{2} \mathrm{FC} \mid$ were analyzed at the functional level for GO and KEGG pathway enrichment using the STRING Enrichment App of the Cytoscape software (https://cytoscape.org/), following Shannon et al. (2003), with $P$ value $<0.05$ as the cutoff criterion. The enrichment analyses were depicted using $R$ programming (https://www.r-project.org/; version 3.6.1 [2019-07-05]) ggplot tools from the Biobase packages (https://CRAN.R-project.org/package = ggplot2) of the Bioconductor project (Huber et al., 2015).

\subsection{SARS-CoV-2 protein and drug interaction (PDI) network analysis}

The PDI network was constructed with STITCH: protein/compound query for the three groups A, B, and C comprising top 44 COVID-19 related genes, 255 DEGs among COVID-19 case compared to uninfected control, and 363 DEGs among ACE2 induced cell line treated with and without SARS-CoV-2, using STRING App of the Cytoscape software, version 3.7.2 (https://cytoscape.org/), according to Shannon et al. (2003); the approved and experimental repurposable drugs against COVID-19 were included to create the PDI network (Guy et al., 2020; Li et al., 2020).

\subsection{Possible repurposable drugs in SARS-CoV-2 and human protein interaction}

The host-virus gene interaction in COVID-19 case and SARS-CoV-2 infected cell line were combined with PDI network to demonstrate potential repurposable therapeutics in the treatment of COVID-19, using all possible combinations of DEGs significance level: case significant and cell line insignificant, case insignificant and cell line significant, both case and cell line significant (case less than cell line), both case and cell line significant (case greater than cell line), and both case and cell line insignificant.

\section{Results And Discussion}

The putative physiological response to SARS-CoV-2 infection from virus entry to release from the host, in the present study, as expressed from COVID-19 case and SARS-CoV-2 infected cell line is depicted in 
Figure 1. The SARS-CoV-2 has evolved strategies including inhibition of host IFN signaling (by nsp1 to decrease p-STAT1 in infected cells) (Wathelet et al., 2007), PKR-mediated apoptosis (NendoU by evading dsRNA sensors in host cell) (WikiPathways, 2020; Deng et al., 2017), NKRF repression by nsp9 and nsp10 as well as hijacking of ubiquitination by nsp10 with DUB activity (WikiPathways, 2020), to evade the host immune response against viral infection. The current dataset GSE5147507 contain the expression of CDK; N protein mediated restriction of cell cycle by CDK4/6 (Surjit et al., 2006), which was significantly decreased in both COVID-19 case and SARS-CoV-2 infected cell line, and nsp14 mediated upregulation of viral replication and transcription by DDX1 (Wu et al., 2014), which was decreased in both COVID-19 case and SARS-CoV2 infected cell line in the current study. The virus counteract IL6 by inducing DUSP1 (decreased in COVID-19 case, elevated in SARS-CoV-2 infected cell line), a negative regulator of p38 MAPK (decreased in both case and cell line), also reported by Liao et al. (2011). The viroporin E protein activate NLRP3 inflammasome (decreased in case, elevated in cell line) to trigger production of TNF, IL6, and IL1B proinflammatory cytokines causing host-immunopathological conditions: hypercytokinemia, ARDS and multi-organ failure (Josh and Manuel, 2020).

Herein, the DEGs of COVID-19 retrieved from STRING disease query of Cytoscape (https://cytoscape.org/) were designated as group A, whereas the dataset GSE5147507 was analyzed to identify DEGsinCOVID-19 case versus uninfected healthy control categorized into group B, and ACE2induced A549 cell line infected with SARS-CoV-2 versus uninfected A549 cell line recognized as group C (Figure 2). The PPI network constructed with top genes from group $A, B$, and $C$ are depicted in Figure 2(ad). The three study groups, A, B, and C displayed 255, 363, and 98 number of DEGs (Figure 2e) with common expression of CSF2 among all the three groups; TMPRSS2 expression was overlapping between group $A$ and group $B$, while CYP1A1, PLEKHA4, and MXT4 expression were common between group $B$ and group $\mathrm{C}$; group $\mathrm{A}$ and group $\mathrm{C}$ shared $\mathrm{ALB}, \mathrm{APOH}, \mathrm{BATF2}$, ENPEP, IFNB1, TNF, and TNNI3 gene expression. There were 18 genes in two clusters: CSF3, IL1B, IL2RA, and IL6 in group A, FOS, MFN2, MYC, PDGFRB, PPP3R1, TSC1 in group B, and ALDH3A1, ACDH3A2, CAT, CES1, CTSD, EGR1, TNF, and TNFSF10 in group C (Figure 3a).

Apart from the genes involved in SARS-CoV-2 infection from virus entry to release from the host (Figure 1), it elicits host innate immune response against the virus through IFN and chemokine signaling (Figure 3b-d). The cells respond to viral PRRs by downstream expression of IRFs (particularly, IRF3 downregulated in case and cell line, IRF9 downregulated in case, and IRF7 upregulated in cell line) and NFKB (downregulated in case; upregulated in cell line) to induce IFN-I and IFN-II signaling. The COVID-19 case study depicted failure to express significant IFN-I transcriptional response due to the absence of IFNA, IFNB, and ISRE, although other genes related to type I and type II IFN signaling were significantly induced in the case (Figure 3c); SARS-CoV-2 infection in cell line too showed significant induction of only few genes related to type I and type II IFN signaling. Within the IFN signaling, the IRF9-STAT2 pathway was expressed in both case (mostly downregulated) and cell line (mostly upregulated) eliciting genes responsible for restricting viral replication (IFI6, IFIT1, IFIT2, IFIT3, IFITM1, IFITM3, OAS1, OAS3, OAS2, MX1, MX2, RSAD2, OASL) and activating apoptosis (XAF1, IRF2, IRF7) (Figure 3d). The SARSCoV-2 viral replication restricted the expression of ISG15 (both case and cell line in the GSE5147507 
dataset analyzed), that play a role in the induction of myriad of antiviral interferon-stimulated genes (Schoggins and Rice, 2011). The ISG20 (in the current dataset GSE5147507), downregulated in COVID19 case, acts as IFN-mediated ssRNA antiviral exoribonuclease, is related to IFNG signaling. Despite virus replication (strong expression in case compared to cell line), the host response to SARS-CoV-2 was ineffective to instigate a strong IFN-I and -II pathways whereas concurrently inducing elevated levels of chemokines required to engage effector cells. The differentially decreased genes that are involved in inflammasome activation and activity include NLRP3, CASP5, IL1A, IL1B, IL18RAP, and IL1R2, and those involved in chemokine signaling for recruiting innate immune cells to the epithelium include CCL2, CCL3, and CCL4, while the most significant ISGs in SARS-CoV-2 infection include IFI6, IFI44L, IFI27 and OAS2 (Mick et al., 2020).

The present dataset featured a wide array of gene expression of chemokine subfamilies (Figure $3 \mathrm{~b}$ ). The most significantly expressed CCL chemokines were CCL 4 and CCL23 with $\log _{2}$ FC values 2.28 and -8.9, respectively in COVID-19 case compared to healthy control group. CCL4 and CCL26 were expressed most significantly with $\log _{2} \mathrm{FC} 2.96$ and -2.76 , respectively along with CCL3 (disease score $=1.8$; $\log _{2} \mathrm{FC}$ 1.1) in SARS-CoV-2 infected- compared to uninfected cell line. In addition to the expression of monocyte associated CCL8 in COVID-19 case, CCL2 (disease score $=1.66$ ) was also expressed significantly in both case and cell line. CCR7 in COVID-19 case ( $\left.\log _{2} F C-11.08\right)$, and CCR6 in both case $\left(\log _{2} F C-6.74\right)$ and cell line $\left(\log _{2} \mathrm{FC} 3.22\right)$ were the most significantly expressed chemokine receptors. Among $\mathrm{CXC}$ subfamily, CXCR4 (disease score $=1.85 ; \log _{2} \mathrm{FC}-6.8$ in case and 2.42 in cell line, $\mathrm{CXCL} 10$ (disease score $=1.79$; $\log _{2} \mathrm{FC} 2.07$ in cell line), CXCL11 ( $\log _{2} \mathrm{FC}-1.62$ in case and 5.25 in cell line) and CXCL2 $\left(\log _{2} \mathrm{FC}-7.7\right.$ in case and 4.24 in cell line) were significant. CXCR4 is involved with the binding of CD4 to support HIV entry into cells and highly expressed in cancer; CXCL11 is dominant ligand for CXCR3 (CXCL3 $\log _{2} \mathrm{FC}-$ 7.15 in case and 2.98 in cell line) and is induced by IFNG while CXCL2 is expressed at the inflammatory site and may inhibit hematopoietic progenitor cell proliferation (GeneCards, 2020). The current dataset expressed CX3CL1 $\left(\log _{2} \mathrm{FC}-5.73\right)$ in COVID-19 case and CX3CR1 $\left(\log _{2} \mathrm{FC}-1.32\right)$ in cell line. The CX3CL1 plays role in cancer, atherosclerosis, AIDS, and inflammatory diseases; CX3CR1 gene is involved in adhesion, migration of leucocytes, and acts as a co-receptor for HIV1 (GeneCards, 2020).

The present study alongside the other reports (Schett et al., 2020) indicate SARS-CoV-2 infection, marked by low IFN-I and IFN-II signaling, induce profuse release of proinflammatory cytokines (TNF, IL1B, IL6, IL8, CSF2, CSF3), chemokines (CCL2, CXCL10, CCL3) and T cell-derived IL17 triggering immunopathogenicity with multi-organ damage, even while the immune response seeks to suppress and eradicate the virus. The COVID-19 severity dependent gene expression is depicted in Figure 3e. Among the plasma cytokines (IL2, IL4, IL6, IL10, IL17, TNFA and IFNG) related to COVID-19 severity and progress, the IL10 level was higher in severe compared to mild patients, IL10 was positively correlated with IL6 level, leucocyte count and neutrophil count, but not with lymphocytes and monocytes count (Ouyang et al., 2020).

Functional analyses of statistically enriched genes are represented in Figure 4 and 5 . The most enriched GO and KEGG pathway among known COVID-19 genes were regulation of signaling receptor activity 
(FDR $3.64 \times 10^{-22}$ ) and cytokine-cytokine receptor interaction (FDR $6.01 \times 10^{-22}$ ), respectively (Figure 4a and $5 \mathrm{a}$ ), while among genes downregulated in COVID-19 case were regulation of protein modification $\left(\right.$ FDR $2.03 \times 10^{-5}$ ) and MAPK signaling (FDR $\left.1.23 \times 10^{-5}\right)$ (Figure 4b and 5b), respectively. The top GO and KEGG pathway in downregulated DEGs of SARS-CoV-2 infected cell line were secretion by cell (FDR $1.67 \times 10^{-6}$ ) and metabolism of drugs (FDR $4.66 \times 10^{-8}$ ) (Figure 4c and 5c), respectively, while those among upregulated DEGs were IFN signaling pathway (FDR $\left.1.69 \times 10^{-13}\right)$ and NOD-like receptor signaling (FDR of $3.82 \times 10^{-6}$ ) (Figure $4 \mathrm{~d}$ and $5 \mathrm{~d}$ ), respectively.

The major candidate genes in group A were ACE2 (highest disease score 2.55, significantly downregulated in case) and IL6 (highest degree connectivity 29 , significantly downregulated in case and upregulated in cell line) with cytokine-cytokine receptor interaction as the top enriched pathway. The enriched pathway (Figure 5a), and ACE2 under expression along with IL6 activation are related to angiogenesis and cancer (Feng et al., 2011; Kumari et al., 2016). The DEGs in group B exhibited major candidate genes to be MYC (with highest degree connectivity) and SFN (with highest $\log _{2} \mathrm{FC}$ ) both related to cancer along with FOS, PPP3R1, TSC1, PDGFRB, and MFN2 in PPI network (Figure 2b). The enriched MAPK and PI3K-AKT pathways are important mediators of cellular processes, dysregulation of which is associated with cancer pathogenesis (Raouf et al., 1996; Vara et al., 2004). The key candidate genes in group C DEGs (downregulated) were ALB (highest degree connectivity) and EPHX1 (highest $\log _{2} \mathrm{FC}$ ) (Figure 2C), and upregulated DEGs were TNF with highest degree connectivity and IFNB1 with highest $\log _{2} \mathrm{FC}$ (Figure $2 \mathrm{~d}$ ). The gene families for ALB and TNF are plasma proteins, intracellular and secreted proteins, in addition to cancer-related and candidate cardiovascular disease genes. Similarly, EPHX1 is related to intracellular proteins, cancer-related genes along with potential drug targets, whereas IFNB1 belongs to cancer-related and secreted proteins related gene families (Human Protein Atlas, 2020). Several of the other cancer associated genes (GSTA1, ABCG2, CAT, CTSD, TF, EGR1, TNFSF10) appeared in the network (Figure 2c-d) were linked with transcriptional response to SARS-CoV-2 infected cell lines affecting innate immunity (CHRNB4, CAT, CTSD, IFNB1), and endothelial and vascular inflammation (TNF, ICAM) in COVID-19. These DEGs altogether displayed association with old and new drugs potentially useful in COVID-19 therapy and tested or used in the oncological settings as well (Ciliberto et al., 2020), including rapamycin, chloroquine, lopinavir, ritonavir, ribavirin as appeared in PDI network in our study (Figure 6), remdesivir, tocilizumab and sarilumab as shown in virus-human protein interaction (Figure 1). The PDI network of group A genes showed top scores of 0.985 and 0.923 for rapamycin (degree of interaction: 5) with IL10, IL2A, CD8A, CD40LG, and CSF3 (Figure 6a). Cyclosporine possessed the highest degree of interaction ( $=7$ ) with CD40LG, IL1B, IFNG, CD8A, IL6, CSF3, and IL2RA, with score ranging from 0.453 to 0.897 ) (Figure 6a). IL6 gene had highest degree of interaction ( = 4) with azithromycin, oseltamivir, cyclosporine, and prednisone]. The PDI network of group B genes showed top scores of 0.926 and 0.876 respectively, in PPP3R1-cyclosporine and MYC-doxycycline interactions; rapamycin had highest degree of interaction ( = 5) with TSC1, MYC, PDGFRB, MFN2, and PPP3R1; MYC was commonly interacting gene for doxycycline, rapamycin and cyclosporine (Figure 6b). In group C, TNF-chloroquine and ABCG2-cyclosporine had top interaction scores of 0.969 and 0.955 . The highest degree of interaction ( = 5) was achieved for rapamycin (scores: 0.683-0.884), with ABCG2, GSTA1, TF, ALDH3A1 and 
ALDH3A2, while the gene with highest degree $(=6)$ was ABCG2 having interaction with cyclosporine, rapamycin, ivermectin, ritonavir, $\mathrm{CHQ}$, and macrolides (scores: 0.466-0.955) (Figure 6c). In general, the PDI network showed rapamycin as top interactor with IL6, MYC and ABCG2 genes in group A, B and C, respectively (Figure 6a-c).

Combined with the current findings, several approved or experimental drugs have been repurposed against SARS-CoV-2 including remdesivir (HIV reverse transcriptase inhibitor), anti-influenza favipiravir (RdRp inhibitor), ribavirin (RNA synthesis and capping inhibitor), anti-influenza baloxavir marboxil (capdependent endonuclease), HIV protease inhibitors lopinavir and ritonavir, endocytosis inhibitor antimalarial chloroquine/hydroxychloroquine, antibiotic azithromycin, immunosuppressant cyclosporine (cyclophilin inhibitor), rapamycin (FKBP/mTORC inhibitor), tocilizumab (IL6 receptor antagonist), sarilumab (tocilizumab efficacy dependent), antihelminthic ivermectin (integrase and importin $\alpha / \beta 1$ inhibitor), antiprotozoal/antiviral netazoxanide (inhibitor of viral replication by tizoxanide metabolite), nefamostat/camostat (ACE2 receptor/TMPRSS2 inhibitor), antidepressant fluvoxamine, INF $\beta-1 \mathrm{a}$ inhibitor (CD73 ecto-5'-neucleotidase) are under clinical trials for COVID-19 treatment (Guy et al., 2020; Li et al., 2020; Bellingan et al., 2014; Caly et al., 2020; Grein et al., 2020; Wu et al., 2020; Uno, 2020).

\section{Conclusions}

Overall, the plausible repurposing drugs for COVID-19 treatment include cyclosporine, doxycycline, chloroquine and rapamycin that emerged as top scoring interactors with MYC, PPP3R1, FOS, ABCG2, and TNF in SARS-CoV-2 infection compared to drug (cyclosporine, azithromycin, rapamycin, and oseltamivir) interaction with IL6, CD8A, CD40LG, IL10, and CSF3 known COVID-19 genes. Among a plethora of available repositionable drugs, those with their targets, appearing in this investigation, might be helpful to combat the ongoing COVID-19 pandemic.

\section{Declarations}

Funding: There was no source of funding for this study.

Conflict of interests: There are no potential conflicts of interests for any authors.

Authors' contribution: MM: Retrieved, processed and analyzed data, and wrote the paper; SM: designed the study, discussed and co-wrote the paper.

\section{References}

Blanco-Melo, D., Nilsson-Payant, B. E., Liu, W. C., Uhl, S., Hoagland, D., Moller, R., et al., 2020. Imbalanced host response to SARS-CoV-2 drives development of COVID-19. Cell. 181, 1036-1045.

Bellingan, G., Maksimow, M., Howell., D.C., Stotz, M., Beale, R., Beatty, M., et al., 2014. The effect of intravenous interferon-beta-1a (FP-1201) on lung CD73 expression and on acute respiratory distress 
syndrome mortality: an open-label study. Lancet Respir. Med. 2: 98-107.

Caly, L., Druce, J. D., Catton, M., Jans, D. A., Wagstaff, M., 2020. The FDA-approved drug ivermectin inhibits the replication of SARS-CoV-2 in vitro. Antiviral Res. 178, 104787. DOI:

10.1016/j.antiviral.2020.104787

Ciliberto, G., Mancini, R., Paggi, MG. 2020. Drug repurposing against COVID-19: focus on anticancer agents. J. Exp Clin Canc Res. 39, 86. DOI: 10.1186/s13046-020-01590-2

Deng, X., Hackbart, M., Mettelman, R. C., O’Brien, A., Mielech, A.M., Yi, G., et. al., 2017. Coronavirus nsp15 mediates evasion of dsRNA sensors and limits apoptosis in macrophages. PNAS. 114, E4251-E4260.

Doncheva, N. T., Morris, J. H., Gorodkin J, Jensen, L., 2019. Cytoscape stringapp: network analysis and visualization of proteomics data. J. Proteome Res. 18, 623-632.

Feng, Y., Ni, L., Wan, H., Fan, L., Fei, X., Ma, Q., et al., 2011. Overexpression of ACE2 produces antitumor effects via inhibition of angiogenesis and tumor cell invasion in vivo and in vitro. Oncol Rep. 26, 115764.

GeneCards. 2020. GeneCards: the human gene database. Available at: https://www.genecards.org/ (Accessed June 22, 2020).

Gordon, D. E., Jang, G. M., Bouhaddou, M., Xu, J., Obernier, K., White, K. M., et al., 2020. A SARS-CoV-2 protein interaction map reveals targets for drug repurposing. Nature. (Published online 30 April 2020). DOI $10.1038 / \mathrm{s} 41586-020-2286-9$

Grein, J., Ohmagari, N., Shin, D., Diaz, G., Asperges, E., Castagna, E., et al., 2020. Compassionate use of remdesivir for patients with severe Covid-19. N. Engl. J. Med. 382, 2327-2336.

Guy, R. K., DiPaola, R. S., Romanelli, F., Dutch, R. E., 2020. Rapid repurposing of drugs for COVID-19. Science 368, 829-830. DOI: 10.1126/science.abb9332

Human Protein Atlas: Version 9.3., 2020. Available at: https://www.proteinatlas.org/ (Accessed June 22, 2020).

Huber, W., Carey, V.J., Gentleman, R., Anders, S., Carlson, M., Carvalho, B. S., et al. Orchestrating highthroughput genomic analysis with Bioconductor. Nature Methods. 2015; 12(2): 115-121.

Josh, R. J., Manuel, A., 2020. COVID-19 cytokine storm: the interplay between inflammation and coagulation. Lancet Respir. Med. 8, E46-E47.

Khalil RA, Morgan, K. G., 1996. Enzyme translocations during smooth muscle activation, In Biochemistry of Smooth Muscle Contraction, 307-318. DOI: 10.1016/B978-012078160-7/50027-5 
Kim, D., Lee, J. Y., Yang, J. S., Kim, J. W., Kim, V. N., Chang, H., 2020. The architecture of SARS-CoV-2 transcriptome. Cell 181, 914-921.

Kumari, N., Dwarakanath, B. S., Das, A., Bhatt, A. N., 2016. Role of interleukin-6 in cancer progression and therapeutic resistance. Tumor Biol. 37, 11553-11572.

Li, G., Clercq, D. E., 2020. Therapeutic options for the 2019 novel coronavirus (2019-nCoV). Nat. Rev. Drug Discov. 19, 149-50.

Liao, Y., Wang, X., Huang, M., Tam, J. P., Liu, D. X., 2011. Regulation of the p38 mitogen-activated protein kinase and dual-specificity phosphatase 1 feedback loop modulates the induction of interleukin 6 and 8 in cells infected with coronavirus infectious bronchitis virus. Virology 420, 106-116.

Mick, E., Kamm, J., Pisco, A. O., Ratnasiri, B. J. M., Calfee, C. S., Castaneda, G., et al. 2020. Upper airway gene expression differentiates COVID-19 from other acute respiratory illnesses and reveals suppression of innate immune responses by SARS-CoV-2.medRxiv. (Posted May 22, 2020).

DOI:10.1101/2020.05.18.20105171

Ouyang, Y., Yin, J., Wang, W., Shi, H., Shi, Y., Xu, B., et al., 2020. Down-regulated gene expression spectrum and immune responses changed during the disease progression in COVID-19 patients. Clin. Infect. Dis. ciaa462. DOI: 10.1093/cid/ciaa462 (Published online April 20, 2020).

Shaha, B., Modia, P., Sagara, S. R. 2020. In silico studies on therapeutic agents for COVID-19: drug repurposing approach. Life Sci. 252, 117652. DOI: 10.1016/j.Ifs.2020.117652

Shannon, P., Markiel, A., Ozier, O., Baliga, N. S., Wang, J. T., Ramage, D., et al., 2003. Cytoscape: a software environment for integrated models of biomolecular interaction networks. GenomeRes. 13, 2498-2504.

Shang, J., Wan, Y., Luo, C., Ye, G., Geng, Q., Auerbach, A., et al. Cell entry mechanisms of SARS-CoV-2. PNAS. 117, 11727-11734.

Schett, G., Sticherling, M., Neurath, M. F., 2020. COVID-19: risk for cytokine targeting in chronic inflammatory diseases? Nat. Rev. Immunol. 20, 271-72.

Schoggins, J. M., Rice, C. M., 2011. Interferon-stimulated genes and their antiviral effector functions. Curr Opinion Virol. 1: 519-525.

Surjit, M., Liu, B., Chow, V. T., Lal, S. K., 2006. The nucleocapsid protein of severe acute respiratory syndrome-coronavirus inhibits the activity of cyclin-cyclin-dependent kinase complex and blocks $S$ phase progression in mammalian cells. J. Biol. Chem. 281, 10669-10681.

Uno, Y., 2020. Camostat mesilate therapy for COVID19. Intern. Emerg. Med. (Published online April 29, 2020). DOI: $10.1007 /$ s11739-020-02345-9 
Vara, J. A. F., Casado, E., Castro, J., Cejas, P., Belda-Iniesta, C., Gonzalez-Baron, M., 2004. PI3K/Akt signalling pathway and cancer. Cancer Treat. Rev. 30, 193-204.

Wathelet, M. G., Orr, M., Frieman, M. B., Baric, R. S., 2007. Severe acute respiratory syndrome coronavirus evades antiviral signaling: role of nsp1 and rational design of an attenuated strain. J Virol. 81, 162011633.

WikiPathways. 2020. Portal: disease/COVID pathways (June 22, 2020). Available at: https://www.wikipathways.org/index.php/Portal:Disease/COVIDPathways (Accessed June 22, 2020).

World Health Organization. WHO coronavirus disease (COVID-19) dashboard. WHO 2020. Available at: https://covid19.who.int/ (Accessed June 26, 2020).

Wu, C. H., Chen, P. J., Yeh, S. H., 2014. Nucleocapsid phosphorylation and RNA helicase DDX1 recruitment enables coronavirus transition from discontinuous to continuous transcription. Cell Host Microbe 16, 462-472.

Wu, R., Wang, L., Kuo, H. C. D., Shannar, A., Peter, R., Chou, P. J., et al., 2020. An update on current therapeutic drugs treating COVID-19. Curr Pharmacol Rep 6, 56-70.

\section{Figures}




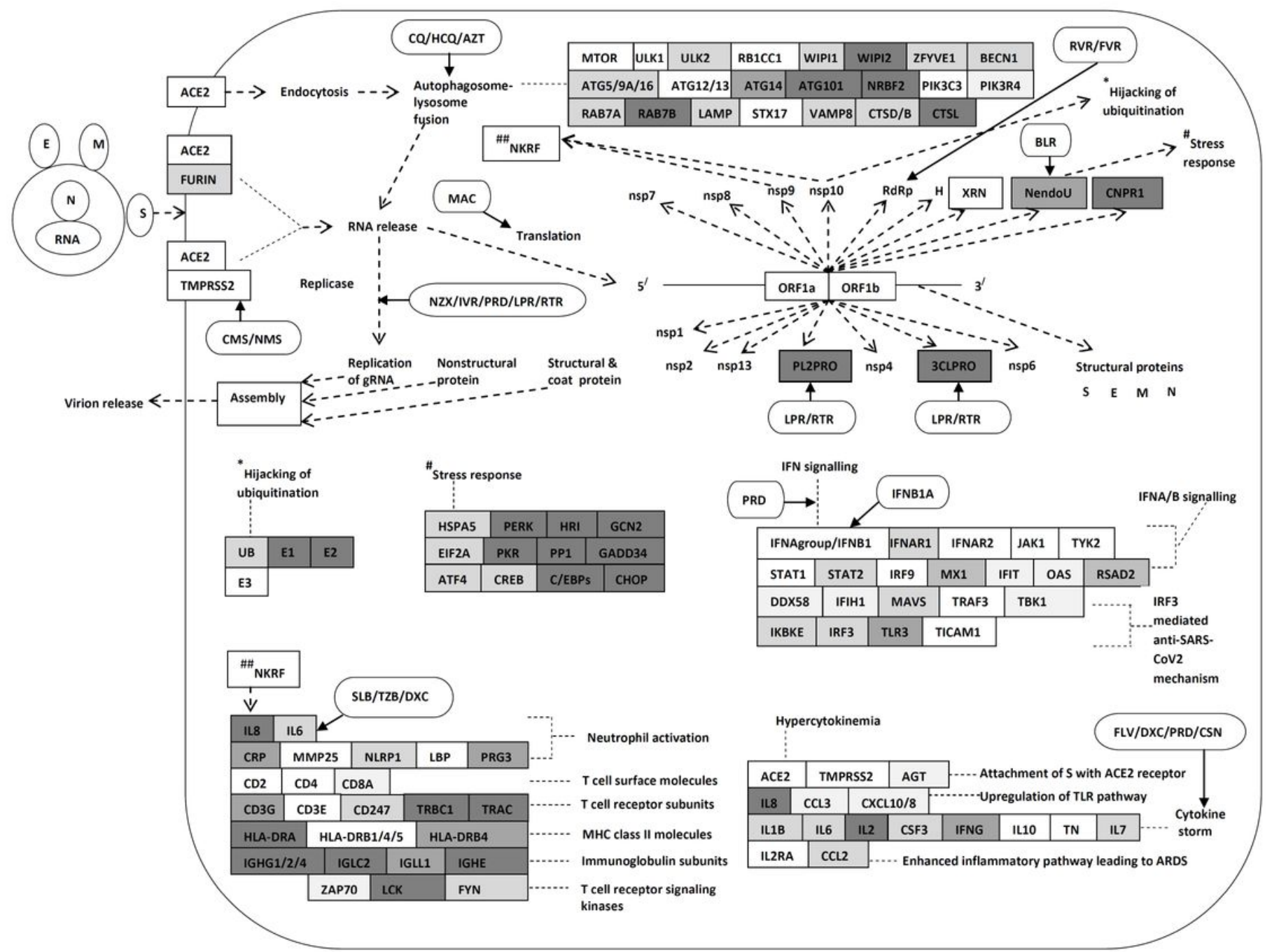

Case significant and cell line insignificant

Case insignificant and cell line significant

Both case and cell line significant (case $>$ cell line)
Both case and cell line significant (case<cell line)

Both case and cell line insignificant

Not found

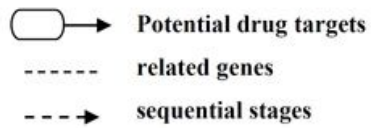

$--\rightarrow$ sequential stages

\section{Figure 1}

Host-virus gene interaction in COVID-19 case and SARS-CoV-2 infected cell line. The RBD of FURIN preactivated SARS-CoV-2 spike protein binds with human ACE2 receptor in association with TMPRSS2 to facilitate viral entry by endocytosis (Guy et al., 2020). Following entry, the virus release genome into the cytosol by autophogosome-lysosomal pathway involving ULK, PI3K, ATG5-ATG12-ATG, and LC3-PE genes. The genomic RNA containing ORF1a and ORF1b is translated to pp1a and pp1ab replicase polyproteins that auto-cleave into $16 \mathrm{nsps}$, to constitute the RTC, which synthesizes minus-stranded RNA (Gordon et al., 2020; Guy et al., 2020). Both full-length and subgenome-length minus strands are synthesized. The structural and accessory proteins are encoded by subgenomic mRNAs positioned at one-fourth of the genome near 3/ end (Shang et al., 2020; Kim et al., 2020). The genomes are then packaged into nucleocapsids and enveloped by budding from smooth intracellular membranes, after which virions release by exocytosis. The SARS-CoV-2 utilizes different strategies (inhibition of host IFN 
signaling, PKR-mediated apoptosis, NKRF repression, and hijacking of ubiquitination) to evade the host immune response while host counteract the SARS-CoV-2 infection through immune response by IFN and chemokine signaling. The repurposable approved and experimental drugs (AZT: azithromycin, BLR: baloxavir, CMS: camostat, CQ: chloroquine, CSN: cyclosporine A, DXC: doxycycline, FLV: fluvoxamine, FVR: favipiravir, HCQ: hydroxychloroquine, IFNB1A: interferon beta 1a, IVR: ivermectin, LPR: lopinavir, MAC: macrolides, NMS: nafamostat: NZX: nitazoxanide, PRD: prednisone, RPN: rapamycin, RTR: ritonavir, RVR: remdesivir, SLB: sarilumab, TZB: tocilizumab) act at several host-virus interaction sites exerting antiSARS-CoV-2 activity. 

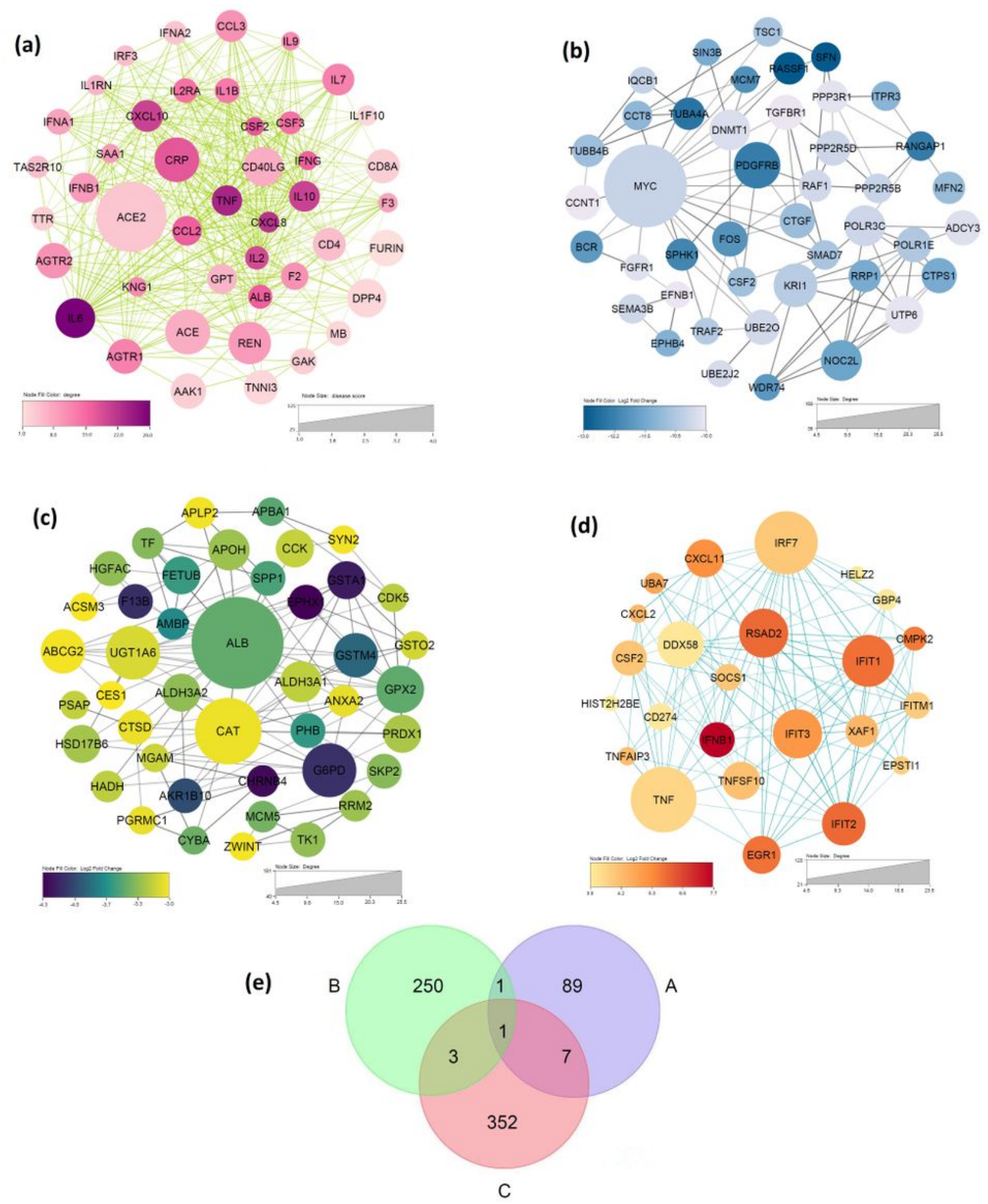

\section{Figure 2}

The DEGs in group A (COVID-19 associated human genes), group B (COVID-19 case versus uninfected healthy control) and group C (ACE2-induced A549 cell line infected with SARS-CoV-2 versus uninfected A549 cell line). PPI network of top genes: (a) group A displaying 44 genes with disease scores (1.0433513.922025) and degree connectivity (1-29), (b) group B (DEG threshold $|\log 2 \mathrm{FC}|)>10$ ) showing $100 \%$ downregulation ( $\mathrm{n}=44)$ with $\log 2 \mathrm{FC}(-13.0$ to -10.0$)$ and degree connectivity (5.0 to 25.0), (c) group C (DEG 
threshold $|\log 2 \mathrm{FC}|)>3$ ) with $64.74 \%$ gene downregulation $(\mathrm{n}=46), \log 2 \mathrm{FC}(-4.3$ to -3.0$)$ and degree connectivity (5.0 to 25.0 ), (d) group C (DEG threshold $|\log 2 \mathrm{FC}|)>3$ ) with $35.26 \%$ gene upregulation $(n=24)$, log2FC (3.0-7.7) and degree connectivity (5.0-23.0); (e) Venn diagram consisting of 255, 363, and 98 DEGs in group $A, B$ and $C$, respectively.

(a)

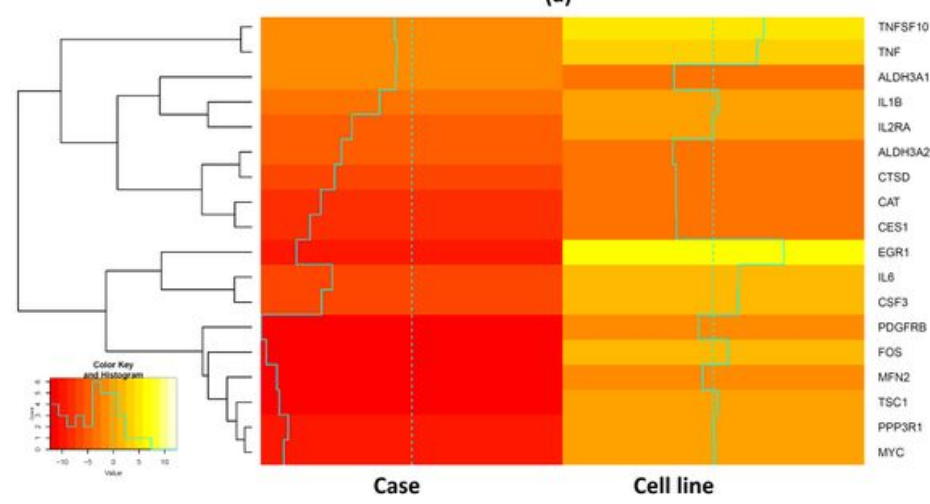

(b)

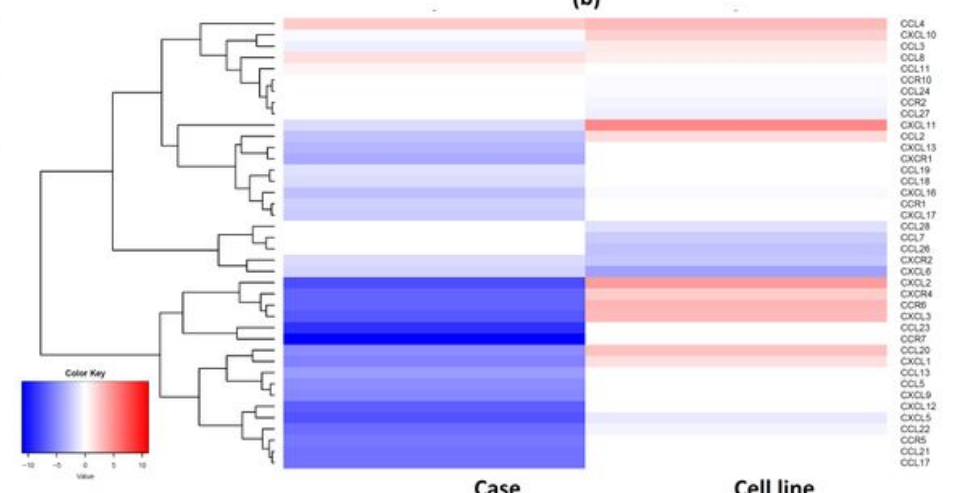

(c)

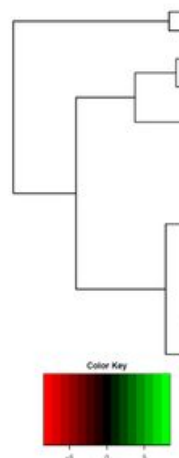

(d)
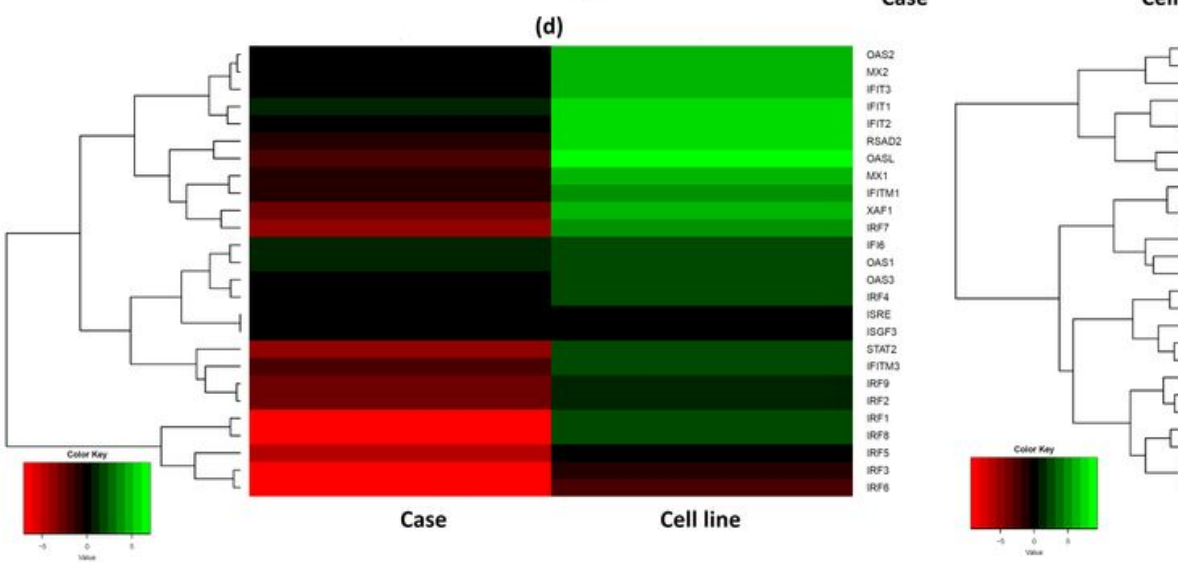

(e)

\section{Figure 3}

Heatmaps of DEGs in COVID-19 case and SARS-CoV-2 infected cell line: (a) key genes of group A, B and C displaying 18 DEGs between lung biopsy sample of COVID-19 case compared to uninfected control and ACE2 transduced lung alveolar A549 cell line infected with or without SARS-CoV-2; (b-d) host innate immune response through chemokine signaling, IFN signaling, and IRF9-STAT2 pathway, (e) COVID-19 severity dependent gene expression (The interleukin-based COVID-19 disease mechanism centered around highly downregulated ILs including IL7, IL6, IL10, IL1B, and IL2RA in case and less significantly upregulated ILs, IL7 and IL6 in cell line. The COVID-19 severity related downregulated genes in case were IL6, IL7, IL10, IL1B, IL2RE, TNF, CCL2, CSF3, PIAS1, RPS6, HSPA8, JUNB, NFATC3, JAK1, and AHR, while in 
cell line the mostly expressed upregulated genes related to COVID-19 severity were JUNB, IL6, TNF, CXCL10, IL7, and CCL2; the downregulated genes were RPS6, HSPA8, and NFATC3).

(a)

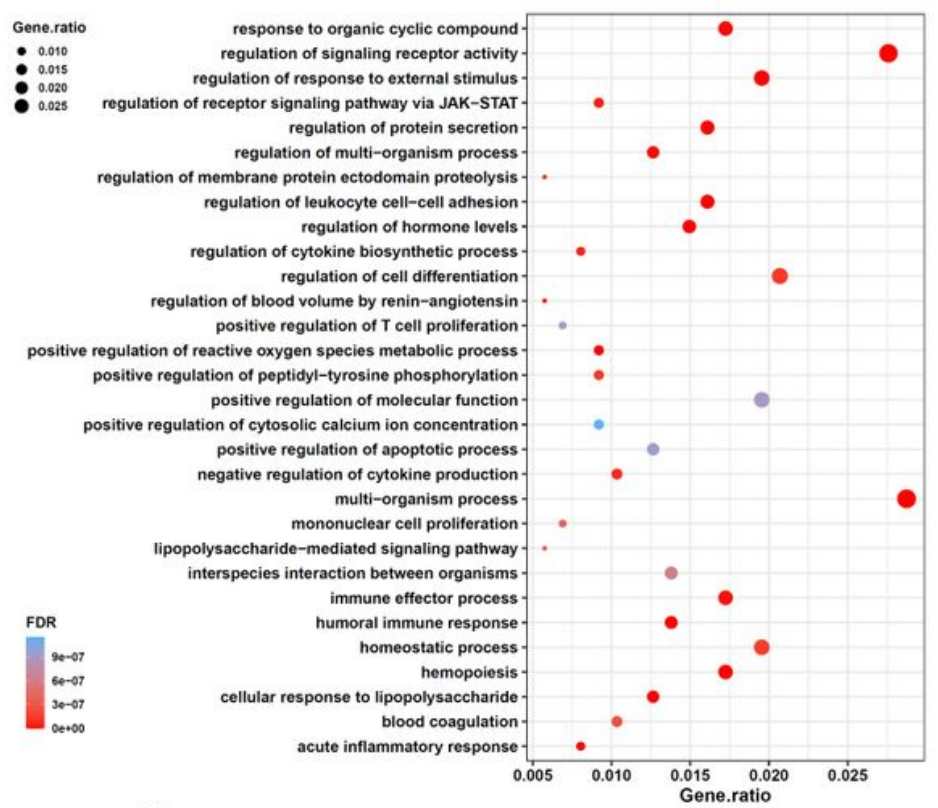

(c)

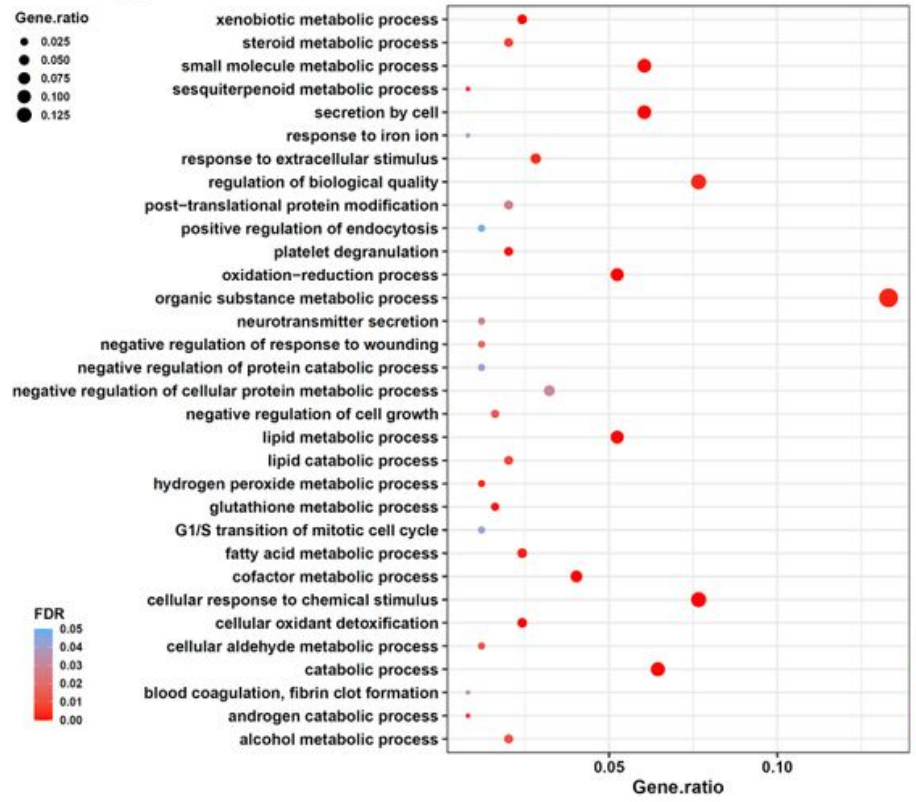

(b)

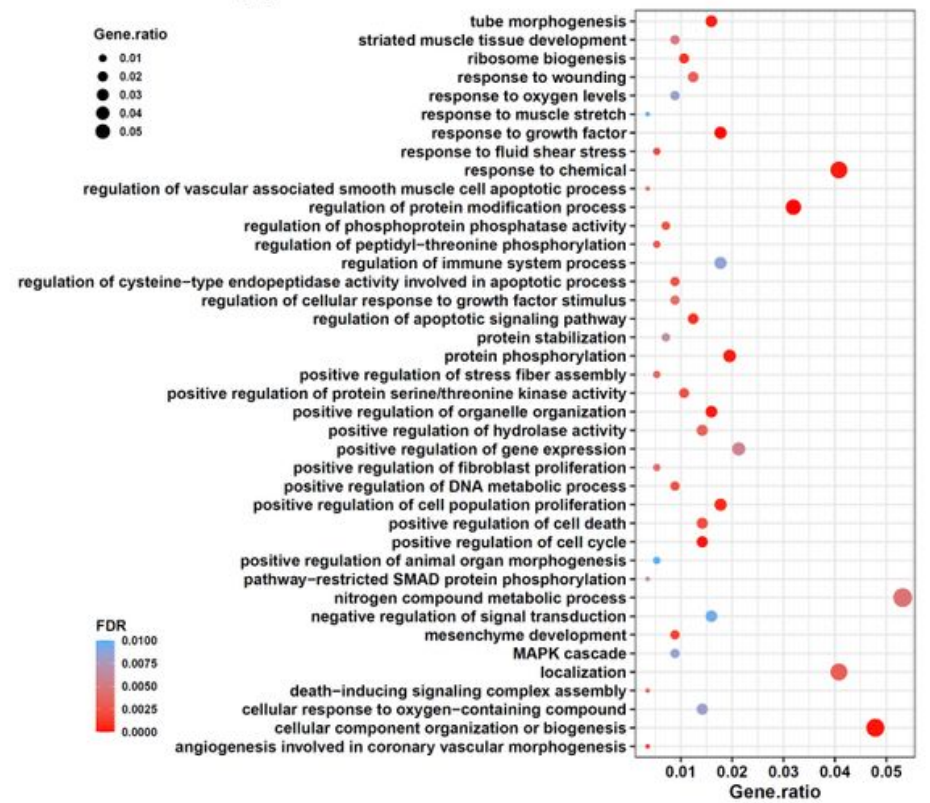

(d)

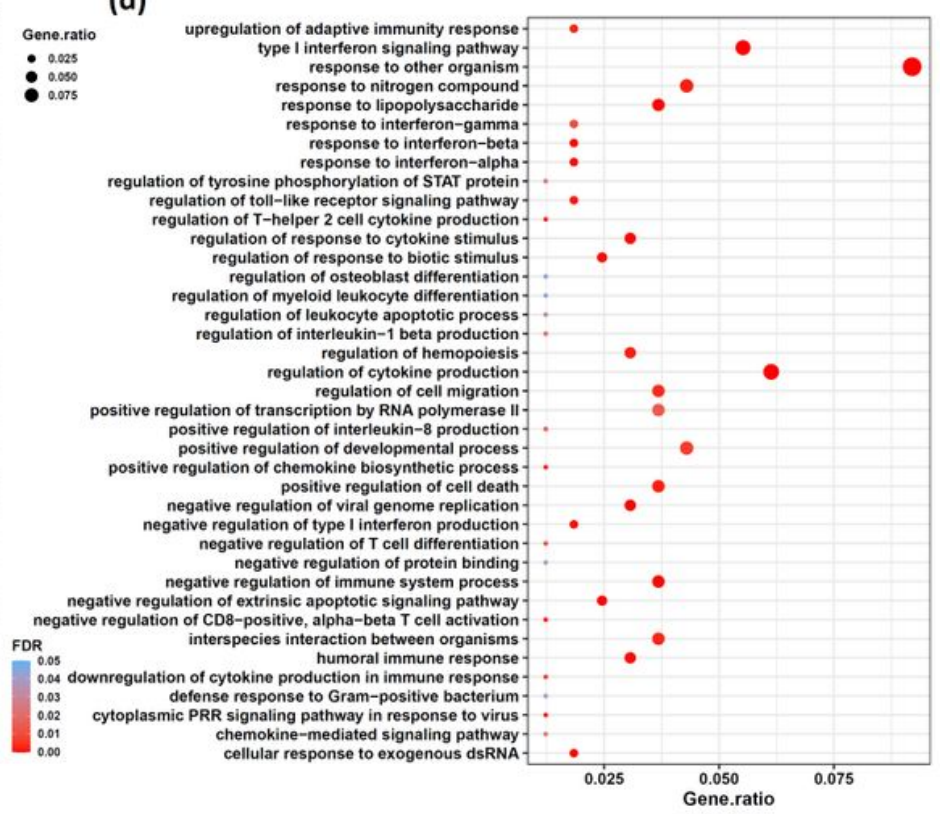

\section{Figure 4}

Functional enrichment analysis of DEGs. Gene Ontology: (a) known COVID-19 genes, (b) COVID-19 case compared to uninfected healthy control, (c) SARS-CoV-2 infected versus uninfected cell line downregulated genes, (d) SARS-CoV-2 infected versus uninfected cell line upregulated genes. The top two enriched biological processes were regulation of signaling receptor activity (FDR $3.64 \times 10-22)$ and leukocyte cell-cell adhesion (FDR $1.44 \times 10-13$ ) among known COVID-19 genes (Figure 4a); regulation of protein modification process (FDR $2.03 \times 10-5)$ and response to growth factor (FDR $5.01 \times 10-5)$ among DEGs downregulated in COVID-19 case (Figure 4b); secretion by cell (FDR $1.67 \times 10-6)$ and cellular 
oxidant detoxification (FDR $1.57 \times 10-5$ ) among downregulated DEGs of SARS-CoV-2 infected cell line (Figure 4c); while those were type I interferon signaling pathway (FDR $1.69 \times 10-13$ ) and response to other organism (FDR $6.96 \times 10-13$ ) among upregulated DEGs of SARS-CoV2 infected cell line in group C (Figure $4 d)$.

(a)

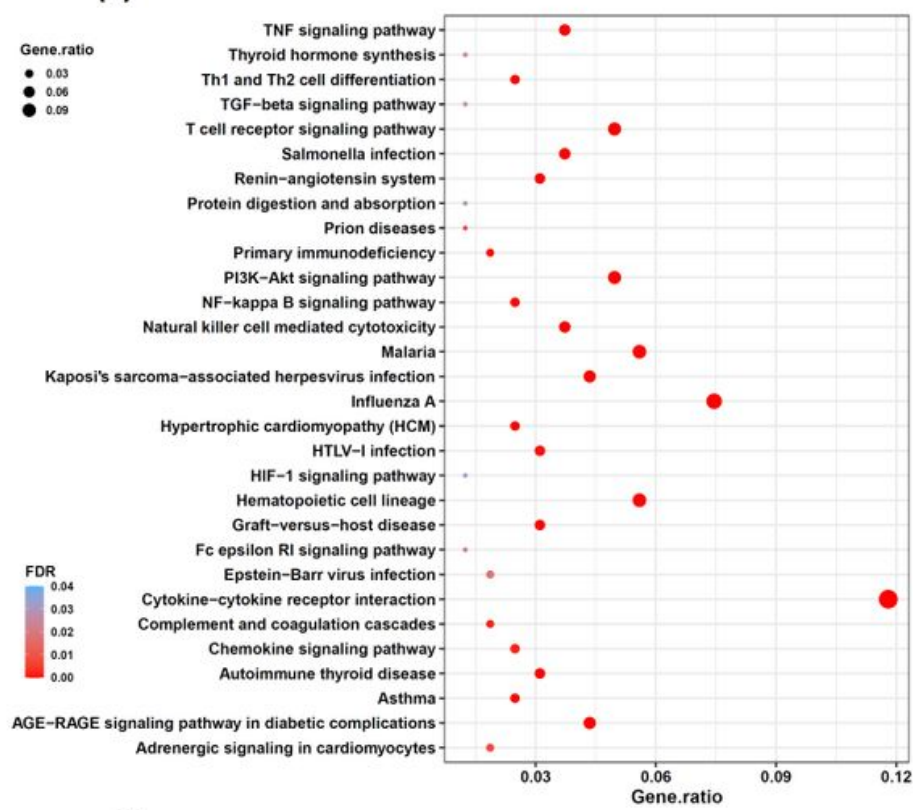

(c)

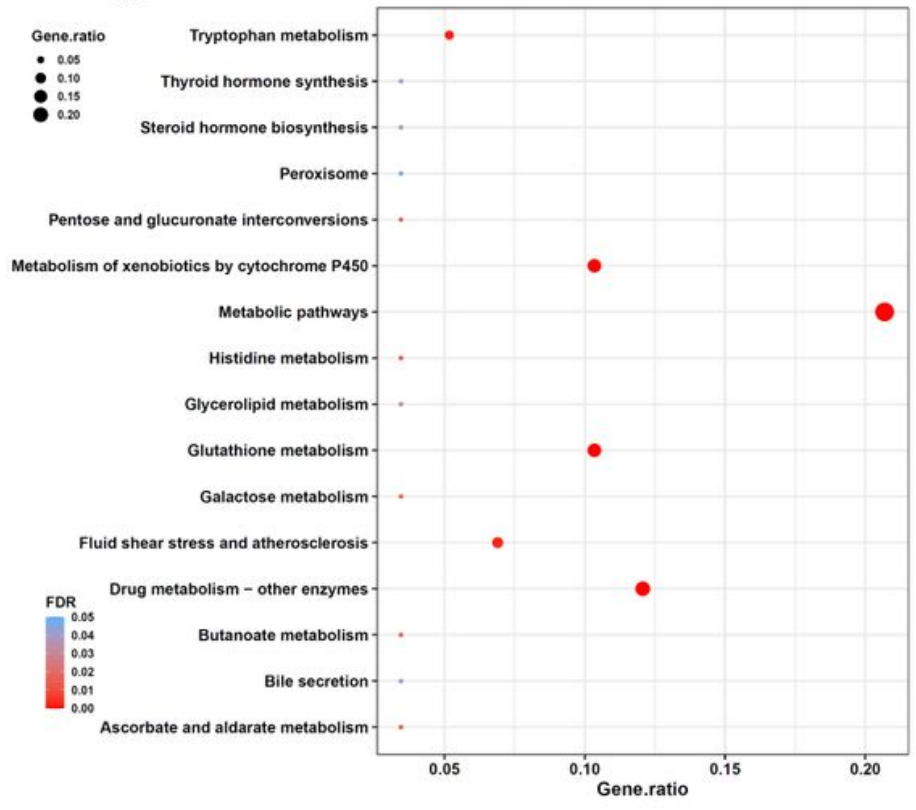

(b)

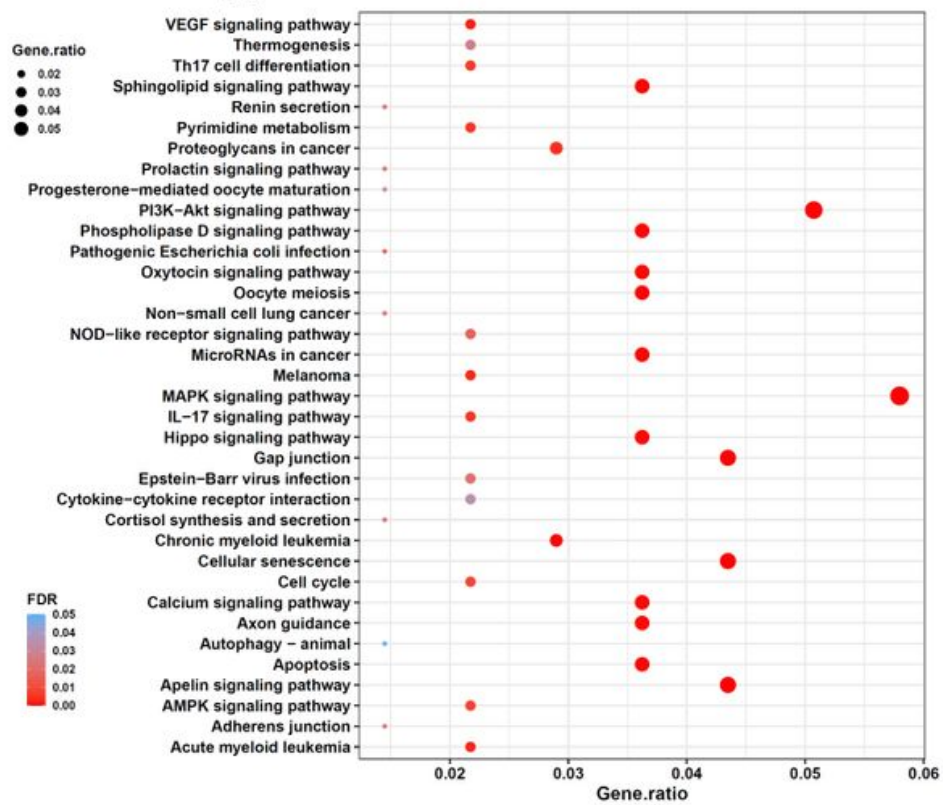

(d)

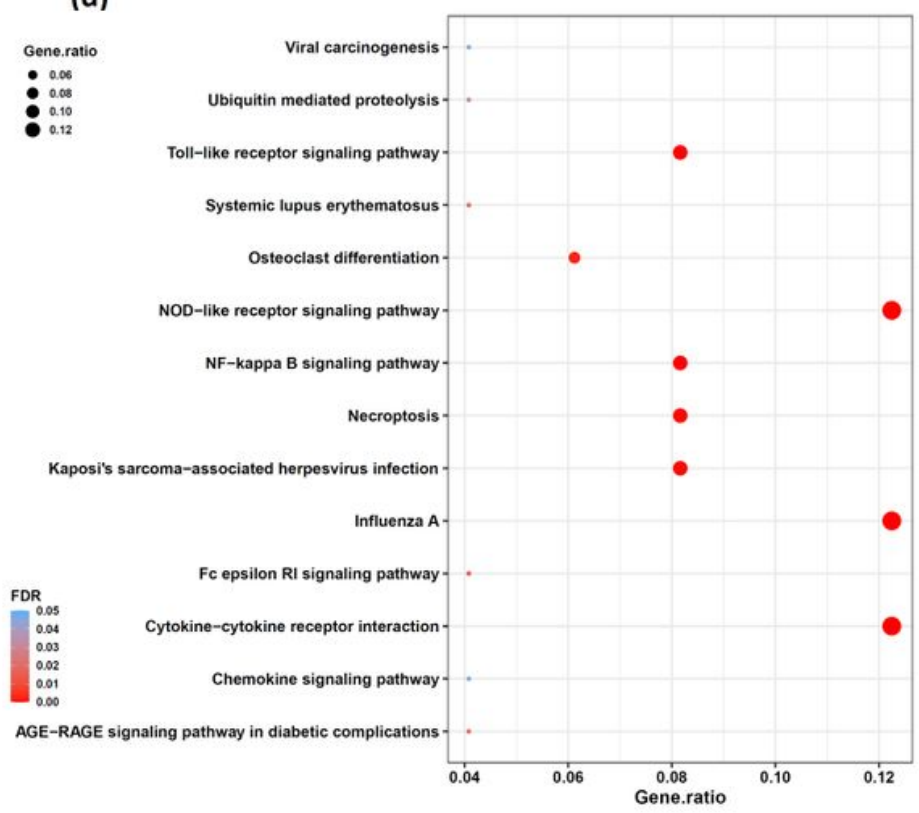

\section{Figure 5}

Functional enrichment analysis of DEGs. KEGG pathway: (a) known COVID-19 genes, (b) COVID-19 case compared to uninfected healthy control, (c) SARS-CoV-2 infected versus uninfected cell line downregulated genes, (d) SARS-CoV-2 infected versus uninfected cell line upregulated genes. The group A genes were enriched with top two KEGG pathways in cytokine-cytokine receptor interaction (FDR $6.01 \times$ 10-22) and malaria (FDR $1.1 \times 10-13$ ) (Figure 5a). Group B genes exhibited mostly enriched pathways 
including MAPK signaling (FDR $1.23 \times 10-5)$ and PI3K-AKT signaling (FDR $1.8 \times 10-4)$ (Figure 5b). Among downregulated DEGs in group $C$, pathways enriched were metabolism of drugs, glutathione, xenobiotics, tryptophan, and histidine, etc. (Figure $5 \mathrm{c}$ ). Among upregulated DEGs in group $\mathrm{C}$, the majorly enriched pathways were NOD-like receptor signaling, influenza A (both with FDR of $3.82 \times 10-6$ ) and cytokinecytokine receptor interaction (FDR $1.47 \times 10-5)$ (Figure 5d).

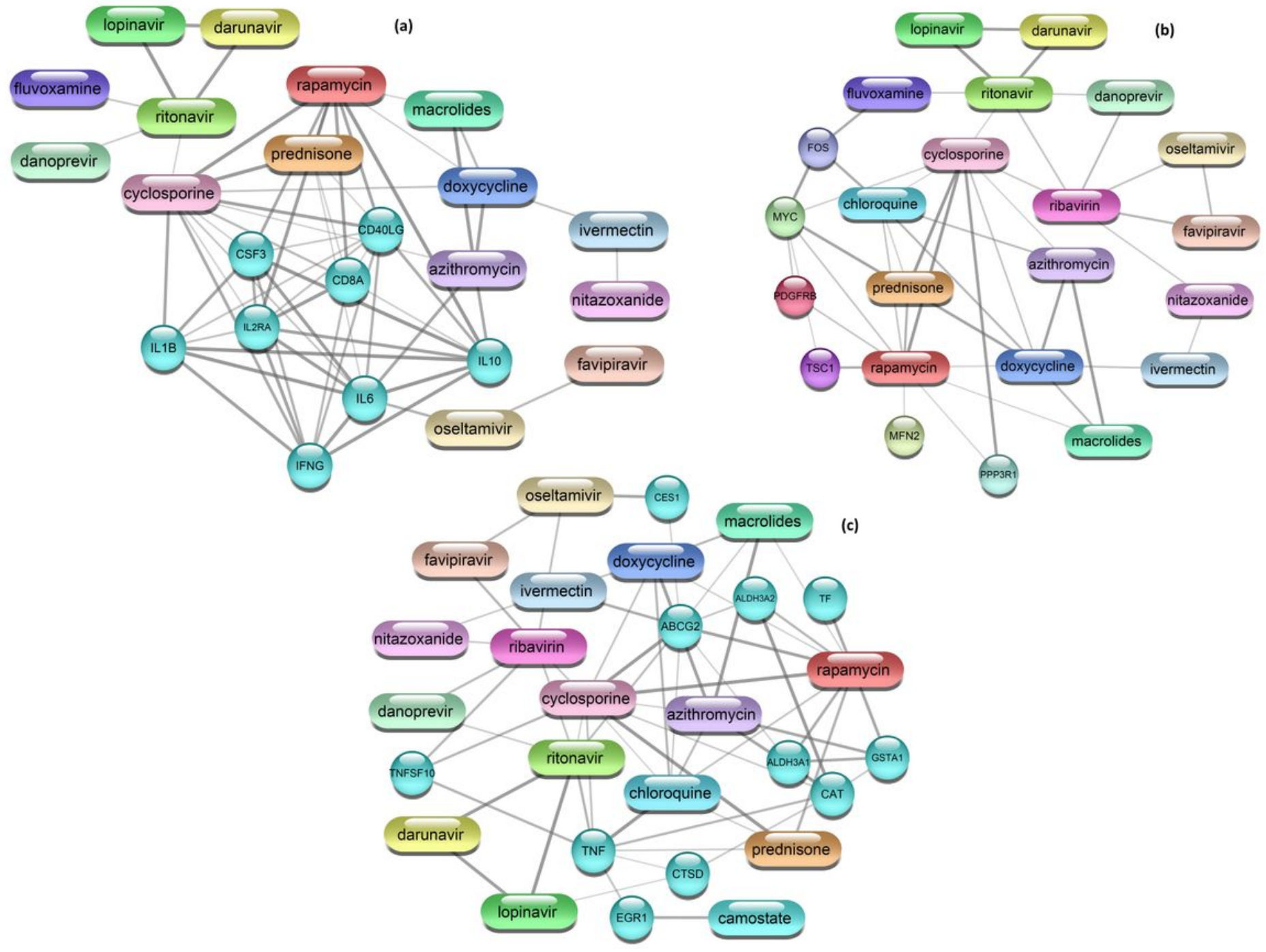

Figure 6

PDI network of repurposable drugs versus top genes from (a) group A, (b) group B, (c) group C. 